Sādhanā Vol. 39, Part 2, April 2014, pp. 345-361. (C) Indian Academy of Sciences

\title{
Fractional M-band dual tree complex wavelet transform for digital watermarking
}

\author{
HARPAL SINGH $^{1, *}$, LAKHWINDER KAUR ${ }^{2}$ \\ and KULBIR SINGH ${ }^{3}$
}

1Rayat-Bahra Institute of Engineering and Bio-technology, Kharar 140 104, India
2University College of Engineering, Punjabi University, Patiala 147 002, India
${ }^{3}$ Thapar University, Patiala 147 004, India
e-mail: sharpal2013@gmail.com; mahal2k8@yahoo.com; ksingh@thapar.edu

MS received 3 May 2013; revised 27 August 2013; accepted 31 August 2013

\begin{abstract}
In this paper, a novel digital watermarking scheme using fractional M-band dual tree complex wavelet transform (Fr-M-band-DT-CWT) is proposed. High frequency channels have wide bandwidth and low frequency channels have narrow bandwidth. These characteristics are suitable for analysing low frequency signal, but not for relatively high frequency signal. The images often contain many edges, which may cause rich middle and high frequency components in the 2-band wavelet domain. Therefore, the ordinary 2-band dual tree complex wavelet transform (DTCWT) is not well-suited for analysing the image. So, the M-band DT-CWT with the FrFT called Fr-M-band-DT-CWT is proposed in this paper to address this problem. Further, we integrate the Fr-M-band-DT-CWT with singular value decomposition (SVD) in order to enhance the performance. Experimental results of the proposed watermarking scheme are compared with the previously available watermarking algorithms, fractional Fourier transform (FrFT), fractional wavelet transform (FrWT). Further, the proposed watermark extraction scheme is also tested on different attacks. The results of the present investigations show that the proposed watermarking scheme is superior as compared to other existing watermarking schemes.
\end{abstract}

Keywords. Fractional M-band dual tree complex wavelet transform (Fr-M-band-DT-CWT); fractional fourier transform (FrFT); fractional wavelet transform (FrWT); multiresolution watermarking.

\section{Introduction}

A digital watermark is a code carrying information about the copyright owner, the creator of the work, the authorized consumer and whatever is needed to handle the property rights associated with any given piece of information. The watermark is intended to be permanently embedded

*For correspondence 
into the digital data so that authorized users can easily read it. At the same time, the watermark should not modify the content of the work but slightly (it should be unperceivable or almost unperceivable by human senses), and it should be virtually impossible for unauthorized users to remove it. By means of watermarking the work is still accessible, but permanently marked.

Cox et al (1997) have presented the algorithm for watermarking images, and a methodology for digital watermarking that may be generalized to audio, video, and multimedia data. They advocate that a watermark should be constructed as an independent and identically distributed (i.i.d.) Gaussian random vector that is imperceptibly inserted in a spread-spectrum-like fashion into the perceptually most significant spectral components of the data. Xia et al (1997) have added a pseudo-random sequence to the largest coefficients of the detail bands where perceptual considerations are taken into account by setting the amount of modification proportional to the strength of the coefficient itself. Watermark detection is achieved through comparison with the original un-watermarked image. Barni et al (1998) proposed a solution to the problem of copyright protection of multimedia data in a networked environment. It makes possible to tightly associate to a digital document a code allowing the identification of the data creator, owner, authorized consumer, and so on. In this paper, a new watermarking algorithm for digital images is presented: the method, which operates in the frequency domain, embeds a pseudo-random sequence of real numbers in a selected set of Discrete Cosine Transform (DCT) coefficients. Hwang et al (1999) have presented a watermarking scheme employed in spatial domain using hash functions.

Djurovic et al (2001) have proposed fractional Fourier transform based watermarking scheme for the multimedia copyright protection. After decomposing image via FrFT, transformation coefficients are reordering in non-increasing sequence and the watermark is embedded in the middle coefficients. Zhang et al (2003) proposed an image watermarking scheme which is developed by using the tree-based spatial-frequency feature of wavelet transform. Adnan et al (2003) proposed the MPEG-4 compressed domain video watermarking method and its performance is studied at video bit rates ranging from 128 to $768 \mathrm{~kb} / \mathrm{s}$. The spatial spread-spectrum watermark is embedded directly to compressed MPEG-4 bitstreams by modifying DCT coefficients. Dong et al (2003) have proposed the digital image watermarking scheme that is invariant to rotation, scaling, and translation (RST). They embed watermark in the log-polar mappings of Fourier magnitude spectrum of original image, and use the phase correlation between the LPM of the original image and the LPM of the watermarked image to calculate the displacement of watermark positions in LPM domain. Chin-Shiuh et al (2003) have proposed an algorithm for embedding several watermarks into the same original source.

Kundur et al (2004) proposed the robust approach called FuseMark based on the principles of image fusion for copy protection or water marking robust tagging applications. They considered the problem of logo watermarking in still images and employed multiresolution data fusion principles for watermark embedding and extraction. Wang et al (2004) proposed a wavelet-treebased blind watermarking scheme for copyright protection. The wavelet coefficients of the host image are grouped into so-called super trees. The watermark is embedded by quantizing super trees. Yu et al (2006) proposed a digital watermarking embedding and detecting algorithm for image is presented, which uses the chirp signal as a watermark and embeds in the FrFT domain of the image, and the watermark position and the transform order are used as the encryption keys. With the help of the property of the impulse characteristic in the FrFT domain for chirp signal, the watermark can be detected conveniently. Yuxin et al (2006) have proposed invisible watermarking scheme for digital images, where the watermark is embedded using the block based lapped orthogonal transform (LOT). The embedding process follows a spread spectrum watermarking approach. 
Prayoth et al (2007) have attempted to develop image watermarking algorithms which are portable to a variety of applications such as copyright protection, fingerprinting and identification. Jixin et al (2009) proposed that the multipurpose audio watermarking algorithm was based on Vector Quantization (VQ) in DCT domain using the codeword labelling and index-bit constrained method. By using this algorithm, it can fulfill the requirements of both the copyright protection and content integrity authentication at the same time for the multimedia artworks.

Xuehua (2010) proposed the digital watermarking and its application in image copyright protection. Xinge et al (2010) proposed an effective method for copyright protection of digital products against illegal usage, watermarking in wavelet domain has recently received considerable attention due to the desirable multiresolution property of wavelet transform. Tetsuya et al (2011) have proposed the blind watermarking algorithm which does not need to refer any original data in the watermark extraction process. They also discuss the analogy between the proposed watermarking scheme and CT-CDMA communication systems based on complete complementary codes (Tetsuya et al 2011).

The paper is organised as follows. The brief review of digital watermarking techniques was given in section 1. The description about FrFT and M-band DT-CWT are given in sections 2 and 3 , respectively. Section 4 presents the SVD and proposed watermarking technique. Experimental results are presented in section 5. Finally, the conclusions are made based on the results and discussions in section 6 .

The proposed fractional M-band dual tree complex wavelet transform (Fr-M-band-DT-CWT) is the integration of FrFT, M-band wavelet transform (M-band-DWT) and dual tree complex wavelet transform (DT-CWT). The building blocks of Fr-M-band-DT-CWT are discussed in the following sections.

\section{Fractional fourier transform}

The Fourier transform (FT) is undoubtedly one of the most valuable and frequently used tools in signal processing and analysis. FT is the time-frequency representation of the signal. In timefrequency representation, normally a plane is used with two orthogonal axes corresponding to time and frequency. The conventional FT can be visualized as a change in representation of the $\pi / 2$ signal corresponding to a counter clock-wise rotation of the axis by an angle. Two successive rotations of the signal through $\pi / 2$ will result in an inversion of the time axis. If the rotation is needed through an angle $\alpha$, then FT is not applicable. To overcome this problem, concept of FrFT is introduced by Victor Namias (1980). The FrFT is the generalization of FT. The FrFT is also called rotational Fourier transform or angular Fourier transform (McBride \& Kerr 1987) since it depends on a parameter $\alpha$ and can be interpreted as a rotation by an angle $\alpha$ in the time-frequency plane or decomposition of the signal in terms of chirps.

The 1D FrFT of a function $s(t)$ is defined as:

$$
F^{\alpha}[s(t)](x)=\int_{-\infty}^{\infty} s(t) K_{\alpha}(t, x) d t,
$$


where $\alpha$ is the transform order (or angle) and $K_{\alpha}(t, x)$ is the transform kernel and is given by:

$$
K_{\alpha}(t, x)= \begin{cases}\sqrt{1-i \cot \alpha} e^{i \frac{t^{2}+x^{2}}{2} \cot \alpha-i x t \csc \alpha} & \alpha \neq n \pi \\ \delta(t-x), & \alpha=2 n \pi \\ \delta(t-x), & \alpha=2 n \pi \pm \pi,\end{cases}
$$

where $n$ is a given integer. The FrFT of a signal exists under the same conditions in which its FT exists. The inverse FrFT can be visualized as the FrFT with transform order $-\alpha$. The main property of FrFT is that the signal obtained is in purely time domain if transform order $(\alpha)$ is 0 and in purely frequency domain if transform order $(\alpha)$ is $\pi / 2$.

Due to the separability of the transform, two-dimensional FrFT can be obtained by successively taking one-dimensional FrFT along both $x$ and $y$ directions (in continuous as well as discrete case). The fast algorithms have been developed for FrFT by Djurovic et al (2001) and Yu et al (2006) which are also an attractive feature for watermark embedding purposes.

The combined concepts of M-band wavelets and DT-CWT is the M-band-DT-CWT. Hence, the brief discussion about the M-channel filter bank, M-band wavelets and DT-CWT is given in the following section 3 .

\section{M-channel filter bank}

The structure of the classical one-dimensional filter bank is depicted in figure 1. The input signal $x(n)$ is filtered by a set of $\mathrm{M}$ filters $h_{i}(n)$. The desired filter responses are shown in figure 2. The response of the $i^{\text {th }}$ filter occupies only a subband of $[-\pi, \pi]$. Subband signals are downsampled by $\mathrm{M}$ to give the signal $d_{i}(n)$. At the reconstruction side these subband signals are passed through $g_{i}(n)$ and up sampled by $\mathrm{M}$ to get output signal $y(n)$. The filters $h_{i}(n)$ are analysis filters constituting the analysis filter bank and the filters $g_{i}(n)$ are the synthesis filters constituting the synthesis filter bank. Perfect reconstruction of the signal is an important requirement of M-channel filter bank. Filter bank is said to be perfect reconstruction if $y(n)=x(n)$. Under certain conditions, perfect reconstruction filter banks are associated with wavelet frames for $L^{2}(R)$ (Xinge et al 2010; Gopinath \& Burrus 1992). This association is a correspondence between the filters and scaling and wavelet vectors associated with the wavelet frames.

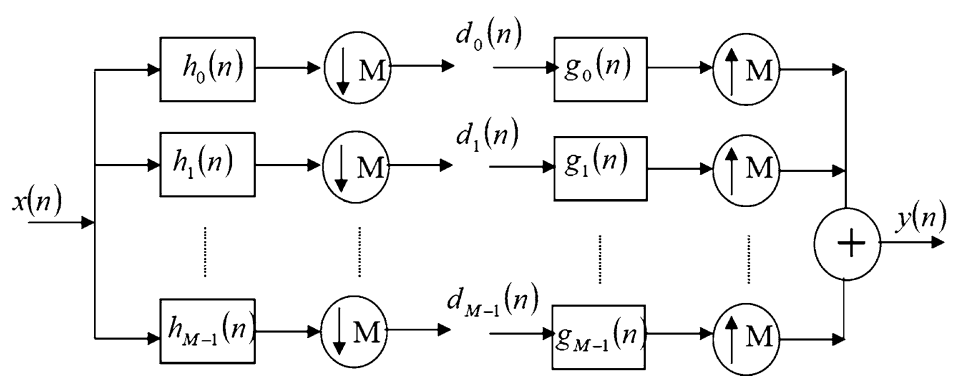

Figure 1. M-Channel filter bank. 


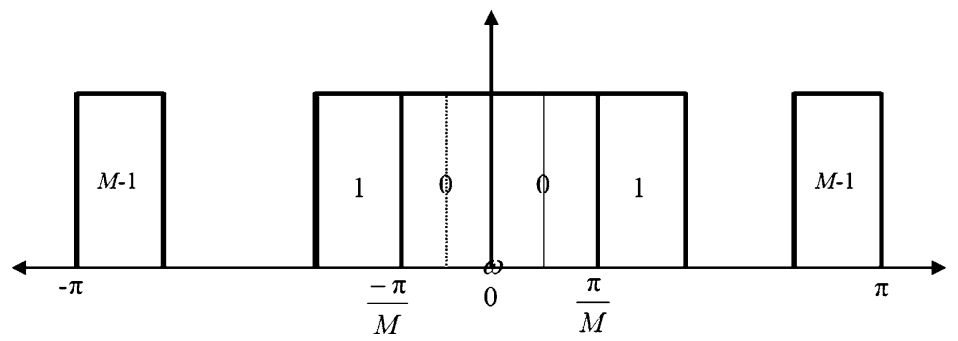

Figure 2. Ideal frequency responses in M-channel filter bank.

\subsection{Dual tree complex wavelet transform (DT-CWT)}

The 1-D DT-CWT decomposes a signal $f(t)$ in terms of a complex shift and dilated mother wavelet $\psi(t)$ and scaling function $\phi(t)$

$$
f(t)=\sum_{l \in Z} s_{j_{0}, l} \phi_{j_{0}, l}(t)+\sum_{j \geq j_{0}} \sum_{l \in Z} c_{j, l} \psi_{j, l}(t),
$$

where $s_{j_{0}, l}$ is scaling coefficient and $c_{j, l}$ is complex wavelet coefficient with $\phi_{j_{0}}$ and $\psi_{j, l}$ complex: $\phi_{j_{0}}=\phi_{j_{0}, l}^{r}+i \phi_{j_{0}, l}^{i}, \psi_{j_{0}}=\psi_{j_{0}, l}^{r}+i \psi_{j_{0}, l}^{i}$. The $\psi_{j_{0}, l}^{r}$ and $\psi_{j_{0}, l}^{i}$ are themselves real wavelets: the complex wavelet transform is a combination of two real wavelet transforms. Figure 3 shows the implementation of 1-D DT-CWT.

2-D DT-CWT can be implemented using separable wavelet transforms like 2-D wavelet transform. Impulse responses of six wavelets associated with 2-D complex wavelet transform is illustrated in figure 4 . These six wavelet subbands of the 2-D DT-CWT are strongly oriented in $\left\{+15^{\circ},+45^{\circ},+75^{\circ},-15^{\circ},-45^{\circ},-75^{\circ}\right\}$ direction and captures image information in that direction. Frequency domain partition of DT-CWT resulting from two level decomposition is shown in figure 5 .

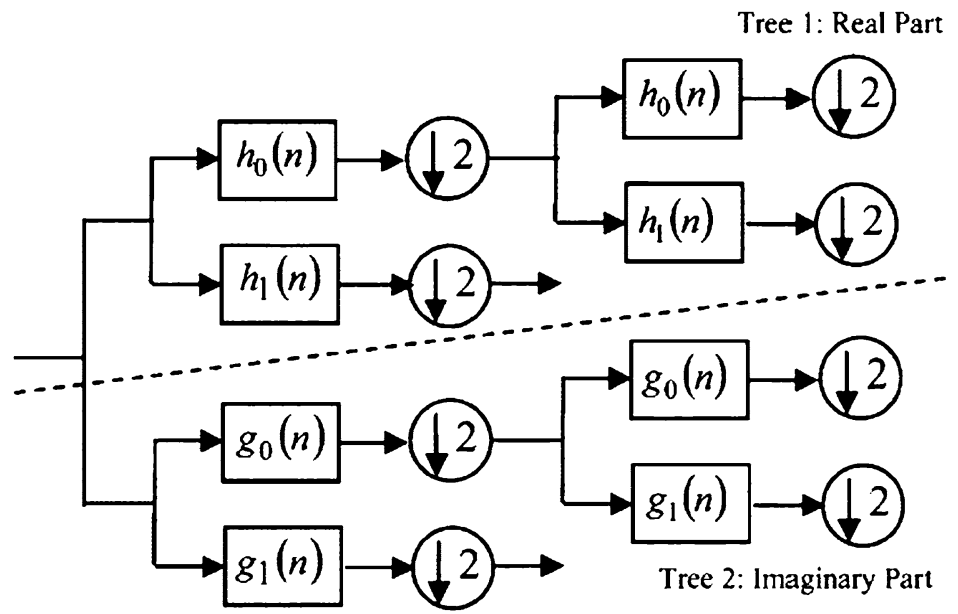

Figure 3. 1-D dual- tree complex wavelet transform. 

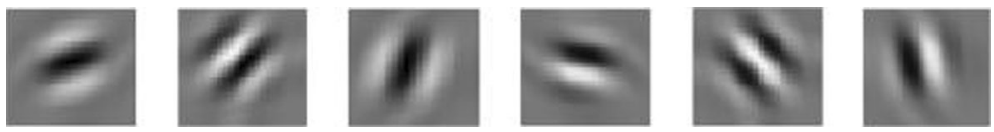

Figure 4. Impulse response of six wavelet filters of DT-CWT.

\subsection{M-band wavelet}

There is a close relationship between M-band wavelets and M-channel filter banks (Gopinath \& Burrus 1992). M-band wavelets are a generalization of the conventional wavelets reported in the literature (Mallat 1989; Rioul \& Veterli 1991). One main disadvantage of using standard wavelets is that they are not suitable for the analysis of high-frequency signals with relatively narrow bandwidth. To overcome this problem, M-band orthonormal wavelet were developed. M-band was developed by generalizing the two band wavelet, which was designed by Daubechies (1988). The M-band orthonormal wavelets give a better energy compaction than two band wavelets by zooming into narrow band high frequency components of a signal (Zou \& Tewfik 1992).

In M-band wavelets there are M-1 wavelets $\psi_{l}(x), l=1,2, \ldots, M-1$, which form the basis functions and are associated with the scaling functions. The M-band wavelet system forms a tight $\left(L^{2}(R)\right)$ frame for the set of square integrable functions defined over the set of real numbers (Gopinath \& Burrus 1992). A function $f(x) \in\left(L^{2}(R)\right)$ is represented by:

$$
f(x)=\sum_{l=1}^{M-1} \sum_{m \in Z} \sum_{n \in Z}\left\langle f(x), \psi_{l, m, n}(x)\right\rangle \psi_{l, m, n}(x),
$$

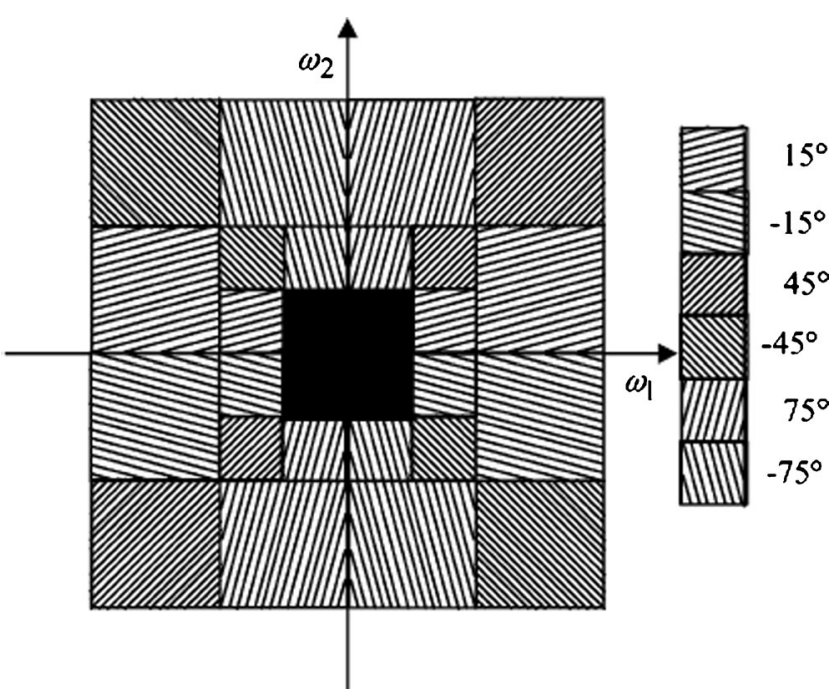

Figure 5. Frequency domain partition in DT-CWT resulting from two level decomposition. 
where $Z$ represents the set of integers and . 〈. $\rangle$ is an inner product. $\psi_{l}(x)$ is scaled and translated to obtain $\psi_{l, m, n}(x)$ functions (Gopinath \& Burrus 1992).

$$
\psi_{l, m, n}(x)=M^{m / 2} \psi_{l}\left(M^{m / 2} x-n\right) \quad l=1,2, \ldots, M-1, m \in Z, n \in Z .
$$

Gopinath \& Burrus (1992) have shown that the wavelet functions $\psi_{l}(x)$ are defined from a unique, compactly supported scaling function $\psi_{0}(x) \in L^{2}(R)$ with support in $[0,(N-1)$ $(M-1)]$ by:

$$
\psi_{l}(x)=\sqrt{M} \sum_{n=0}^{N-1} h_{l}(x) \psi_{0}(M x-n) ; \quad l=1,2, \ldots, M-1 .
$$

The scaling function satisfies the recursion equation:

$$
\psi_{0}(x)=\sqrt{M} \sum_{n=0}^{N-1} h_{0}(x) \psi_{0}(M x-n),
$$

where $h_{0}$ is a scaling filter of length $N=M * K$ ( $K$ is regularity of scaling function), which satisfies the following constraints.

$$
\begin{gathered}
\sum_{n=0}^{N-1} h_{0}(n)=\sqrt{M}, \\
\sum_{n=0}^{N-1} h_{0}(n) h_{0}(n+M i)=\delta(i) .
\end{gathered}
$$

The $(M-1) h_{l}$ filters are also of length $N$ and are called the wavelet filters and satisfy the equation

$$
\sum_{n=0}^{N-1} h_{l}(n) h_{m}(n+M i)=\delta(i) \delta(l-m) \text {. }
$$

\subsection{M-band DT-CWT}

The dual tree complex wavelet (DT-CWT) (Chaux et al 2004; Kokare et al 2004, 2005), which was originally developed using two 2-band DWTs has been extended to M-band DWTs recently and used for image processing. The M-band DT-CWT which employs two M-band DWTs where the wavelets are associated with the two transforms from Hilbert transform pairs.

A typical M-band DT-CWT analysis filter bank for $M=4$ is shown in figure 6 . The filter bank in essence is a set of bandpass filters with frequency and orientation selective properties. In the filtering stage we make use of biorthonormal M-band DT-CWT to decompose the texture image into $\mathrm{M} \times \mathrm{M}$-channels, corresponding to different direction and resolutions. The one-dimensional $M(=4)$-band wavelet filter impulse responses are given by $\psi_{l}$ and their corresponding transfer functions are denoted by $h_{l}$ for $l=0,1,2,3 . \psi_{1}$ is the scaling function (lowpass filter) and other $\psi_{l}$ 's correspond to the wavelet functions (bandpass filters). In this work, we have obtained the $M$ channel 2-D separable transform by the tensor product of M-band 1-D DT-CWT filters. At each level with $\mathrm{M}=4$, the image is decomposed into $\mathrm{M} \times \mathrm{M}(=16)$ channels. Table 1 shows the 4-band dual-tree wavelet filter coefficients used in the experiments. 


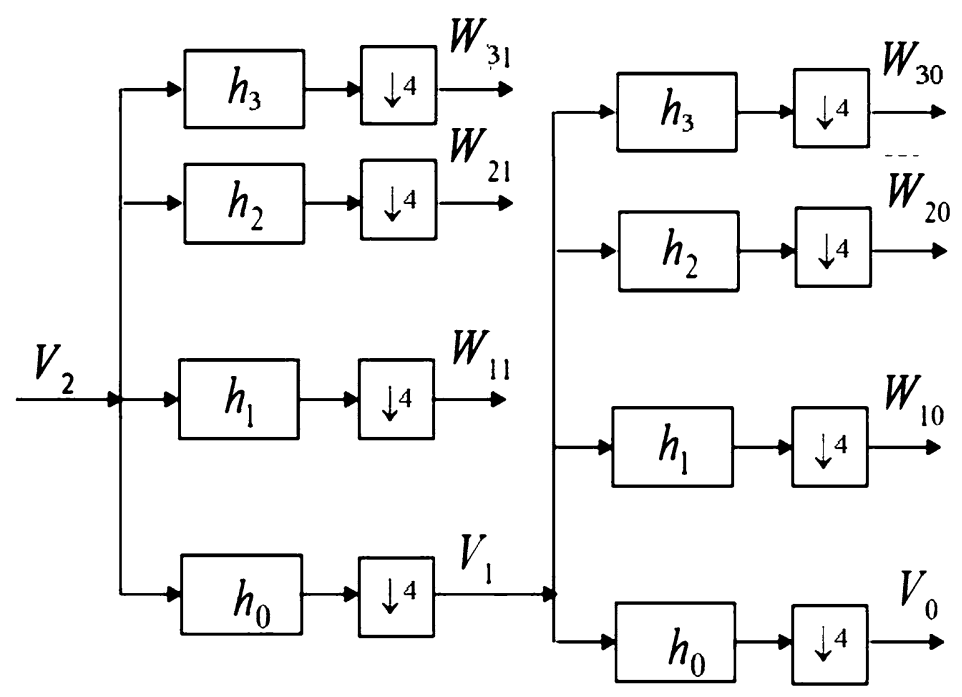

Figure 6. $\mathrm{M}$ - band $(\mathrm{M}=4)$ wavelet filter bank structure.

\subsection{Fractional M-band DT-CWT (Fr-M-band-DT-CWT)}

In this paper, we have proposed the Fr-M-band-DT-CWT by integrating the FrFT and M-band DT-CWT. The algorithm of the proposed Fr-M-band-DT-CWT is given as follows:

\section{Algorithm:}

Input: Image; Output: Fr-M-band-DT-CWT response

1. Load the image and convert into gray scale (if it is RGB).

2. Apply the M-band DT-CWT.

Table 1. Four band dual-tree complex wavelet filter coefficients used in experiments.

\begin{tabular}{llll}
\hline$h_{0}$ & $h_{1}$ & $h_{2}$ & $h_{3}$ \\
\hline 0.030550699 & 0.01990811 & 0.01990811 & 0.030550699 \\
-0.01990811 & -0.030550699 & 0.030550699 & 0.01990811 \\
-0.058475205 & -0.038104884 & -0.038104884 & -0.058475205 \\
-0.038104884 & -0.058475205 & 0.058475205 & 0.038104884 \\
-0.036706282 & -0.168841015 & -0.168841015 & -0.036706282 \\
0.168841015 & 0.036706282 & -0.036706282 & -0.168841015 \\
0.4095423 & 0.544260466 & 0.544260466 & 0.4095423 \\
0.544260466 & 0.4095423 & -0.4095423 & -0.544260466 \\
0.544260466 & -0.4095423 & -0.4095423 & 0.544260466 \\
0.4095423 & -0.544260466 & 0.544260466 & -0.4095423 \\
0.168841015 & -0.036706282 & -0.036706282 & 0.168841015 \\
-0.036706282 & 0.168841015 & -0.168841015 & 0.036706282 \\
-0.038104884 & 0.058475205 & 0.058475205 & -0.038104884 \\
-0.058475205 & 0.038104884 & -0.038104884 & 0.058475205 \\
-0.01990811 & 0.030550699 & 0.030550699 & -0.01990811 \\
0.030550699 & -0.01990811 & 0.01990811 & -0.030550699
\end{tabular}


3. Apply the FrFT on each subband of M-band DT-CWT.

4. Collect the Fr-M-band-DT-CWT responses.

In literature, there are many SVD based watermarking schemes. Some of them are purely SVD based whereas others are hybridized with different transforms. Hybridization is needed in order to enhance the performance and security of the images. Most of the SVD based algorithms are less resilient especially with geometric distortion including resizing, translation etc. In this paper we have attempted integration of the Fr-M-band-DT-CWT with SVD in order to enhance the performance. The brief description about the SVD is given in the following section.

\section{Singular value decomposition}

Let $A$ be a general real (complex) matrix of order $m \times n$. The SVD (Gaurav \& Raman 2011) of $A$ is the factorization (figure 7)

$$
A=U * S * V^{T}
$$

where $U$ and $V$ are orthogonal(unitary) and $S=\operatorname{diag}\left(\sigma_{1}, \sigma_{2}, \ldots, \sigma_{r}\right)$, where $\sigma_{i}, i=1(1) r$ are the singular values of the matrix $A$ with $r=\min (m, n)$ and satisfying $\sigma_{1} \geq \sigma_{2} \geq \ldots \geq \sigma_{r}$. The first $r$ columns of $V$ are the right singular vectors and the first $r$ columns of $U$ are the left singular vectors.

Use of SVD in digital image processing has some advantages. First, the size of the matrices of SVD transformation is not fixed. It can be a square or rectangular. Secondly, singular values in a digital image are less affected if general image processing is performed. Finally, singular values contain intrinsic algebraic image properties.

\subsection{Proposed watermarking scheme}

In this paper, we have used Fr-M-band-DT-CWT and SVD for developing the algorithm.

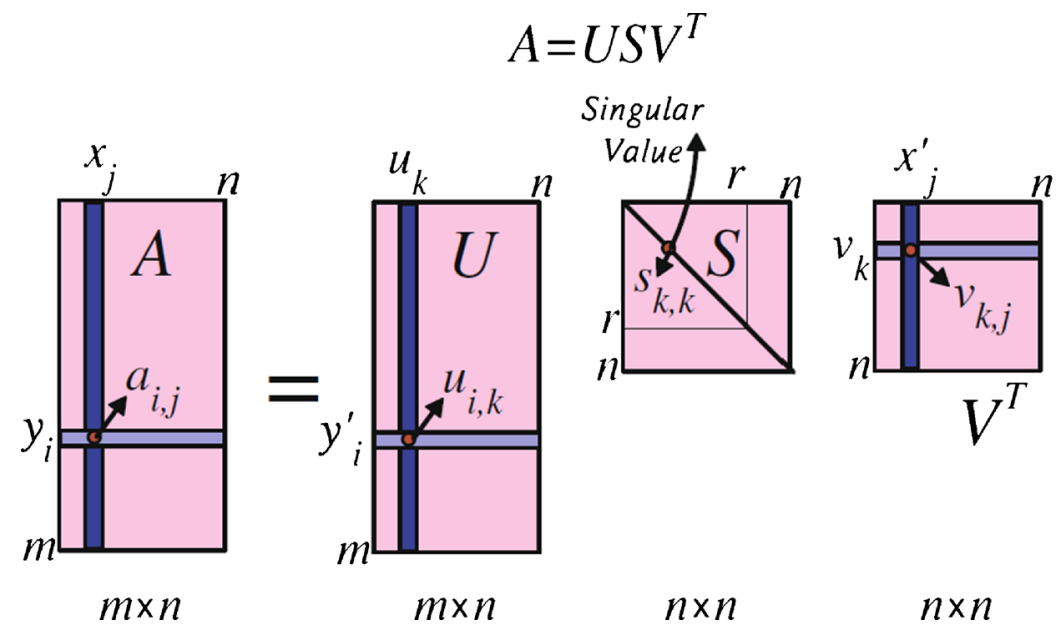

Figure 7. Illustration of the singular value decomposition (SVD). 
The proposed watermarking scheme uses gray-scale images as host and watermark. Let us assume the host and watermark images are represented by $F$ and $W$. The sizes of host and watermark images are $M \times N$ and $M / 4 \times N / 4$, respectively. Block diagram of the proposed watermarking scheme is shown in figure 8 and algorithm for the same is given bellow.

\subsection{Algorithm for watermark embedding}

Input: Host image and watermark; Output: Watermarked image

1. Load the host image and watermark.

2. Apply M-band DT-CWT on host image.

3. Apply FrFT on M-band DT-CWT subbands.

4. Apply SVD on M-band DT-CWT responses and watermark.

5. Embedded the watermark in host image.

6. Apply inverse FrFT on each M-band DT-CWT subbands.

7. Apply inverse M-band DT-CWT transform.

8. Collect the watermarked image.

\subsection{Algorithm for watermark extraction}

Input: Host image, watermarked image and key; Output: Extracted watermark

1. Load the host image and watermarked image.

2. Apply M-band DT-CWT on host and watermarked images.

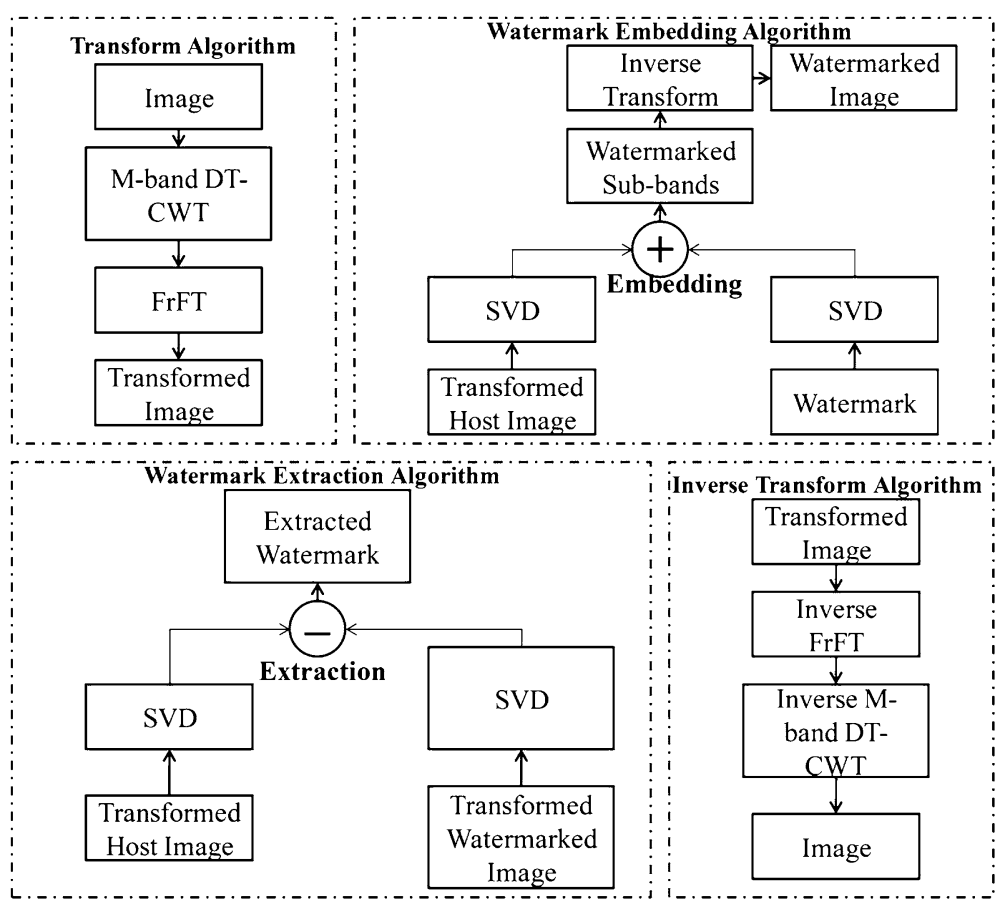

Figure 8. Block diagram of proposed watermark embedding and extraction algorithm. 
3. Apply FrFT on M-band DT-CWT subbands.

4. Apply SVD on M-band DT-CWT responses.

5. Subtract the host image SVD values from watermarked image SVD values using key.

6. Collect the extracted watermark.

\section{Experimental results and discussions}

The robustness of the proposed scheme is demonstrated using MATLAB. Different standard gray-scale images of size $M \times N$ are used as the host image namely, Barbara, Lena and Cameraman. For watermark, gray-scale logo of size $M / 4 \times N / 4$ namely, IEEE Logo, Punjabi University Logo and Punjab Police logo are used. Figure 9 illustrates the selected host images and watermarks for the evaluation of the proposed method. The results of the watermark embedding and extraction are given in the following sections.

The watermarked image quality is measured using Peak Signal to Noise Ratio based on human visual system (PSNR-HVS) (Gaurav \& Balasubramanian 2011). Mathematically, PSNR-HVS between host $(F)$ and watermarked $(\tilde{F})$ images is given by:

$$
P S N R-H V S=10 \times \log \left(\frac{255^{2}}{M S E-H V S}\right),
$$

where MSE - HVS is the mean square error, which is calculated by taking into account of HVS and is given by:

$$
M S E-H V S=K \sum_{i_{1}=1}^{M-7} \sum_{j_{1}=1}^{N-7} \sum_{i=1}^{8} \sum_{j=1}^{8}\left[\left(F(i, j)_{i_{1}, j_{1}}-\tilde{F}(i, j)_{i_{1}, j_{1}}\right) T_{c}(i, j)\right]^{2},
$$

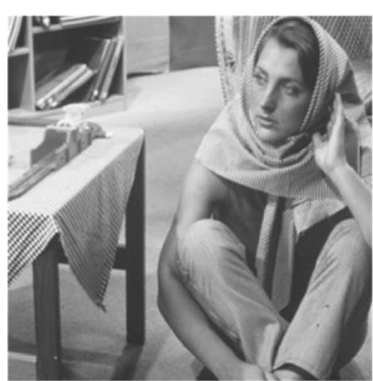

Barbara

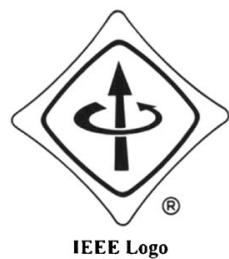

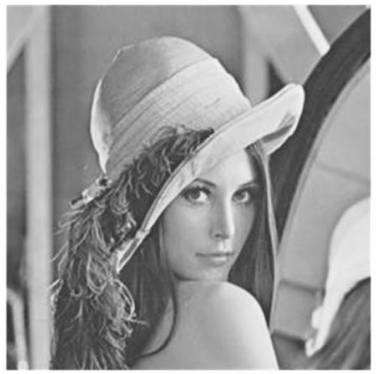

Lena

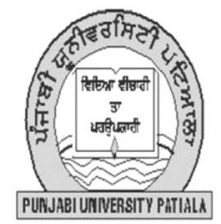

Punjabi University Logo

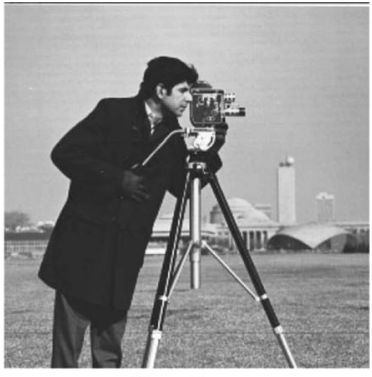

Cameraman

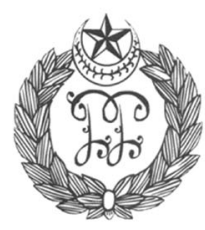

Punjab Police Logo

Figure 9. Sample host images and watermarks. 
Table 2. Peak signal to noise ratio based on human visual system for each host image.

\begin{tabular}{llll}
\hline Method & Barbara & Lena & Cameraman \\
\hline Fr-M-band-DT-CWT & 39.65 & 39.84 & 40.02 \\
\hline
\end{tabular}

where $M$ and $N$ are the number of rows and columns of the images, respectively. In this paper, for calculations, we use $K=1 /[64(M \times N)]$ and $\mathrm{T}_{\mathrm{c}}$ is taken as unity. where, $F(i, j)_{i_{1}, j_{1}}$ and $\tilde{F}(i, j)_{i_{1}, j_{1}}$ is the DCT coefficients of $8 \times 8$ blocks for which the coordinates of its left upper corner are equal to $i_{1}$ and $j_{1}$ for $F$ and $\tilde{F}$, respectively, and Tc is the matrix of correcting factor (Egiazarian et al 2006). The main reason for using PSNR-HVS and MSE-HVS is the limitations of these classical metrics. Classical MSE and PSNR work satisfactorily when the distortion is mainly caused by contamination by additive noise. However, the problem inherent in MSE and PSNR is that they do not take into account the viewing conditions and visual sensitivity with respect to image contents. Hence, PSNR-HVS and MSE-HVS take care of these things while evaluating and perform better than classical metrics. If we observe the host and watermarked images, we cannot find any perceptual degradation according to HVS.

The performance of the proposed watermarking scheme is also evaluated using the correlation coefficient between the original watermark and the extracted watermark as shown in Eq. (14).

$$
\rho(S, \bar{S})=\frac{\sum_{i=1}^{r}\left(S(i)-S_{\text {mean }}\right)-\left(\bar{S}(i)-\bar{S}_{\text {mean }}\right)}{\sqrt{\sum_{i=1}^{r}\left(S(i)-S_{\text {mean }}\right)^{2}} \sqrt{\sum_{i=1}^{r}\left(\bar{S}(i)-\bar{S}_{\text {mean }}\right)^{2}}},
$$

where $S, \bar{S}, S_{\text {mean }}$ and $\bar{S}_{\text {mean }}$ are the original, extracted singular values, mean of original and extracted singular values and $r=\min (M, N)$. The value of $\rho$ lies between $[-1,1]$. If it is equal to 1 then the extracted singular values are just equal to the original one, if it is -1 then the difference is negative for the largest singular values. In this case, the lighter parts of the image become darker and darker parts become lighter i.e., constructed watermark looks like negative thin film.

Table 3. Correlation coefficient $(\rho)$ of extracting watermark with various attacks on Barbara watermarked image. IEEE: IEEE Logo; PU: Punjabi University Logo; PL: Punjab Police Logo.

\begin{tabular}{|c|c|c|c|c|c|c|c|c|c|}
\hline & \multicolumn{3}{|c|}{ Fr-M-band-DT-CWT } & \multicolumn{3}{|c|}{ FrWT } & \multicolumn{3}{|c|}{ FrFT } \\
\hline & IEEE & PU & PL & IEEE & $\mathrm{PU}$ & $\mathrm{PL}$ & IEEE & $\mathrm{PU}$ & $\mathrm{PL}$ \\
\hline No attack & 0.999 & 0.999 & 0.999 & 0.997 & 0.996 & 0.996 & 0.996 & 0.996 & 0.96 \\
\hline Average filtering & 0.999 & 0.998 & 0.999 & 0.330 & 0.276 & 0.265 & 0.414 & 0.359 & 0.318 \\
\hline Median filtering & 0.999 & 0.998 & 0.999 & 0.711 & 0.655 & 0.649 & 0.689 & 0.641 & 0.614 \\
\hline Gaussian noise & 0.985 & 0.983 & 0.974 & 0.254 & 0.229 & 0.249 & 0.247 & 0.231 & 0.283 \\
\hline Salt \& pepper noise & 0.997 & 0.996 & 0.994 & 0.620 & 0.572 & 0.572 & 0.493 & 0.464 & 0.482 \\
\hline Resizing & 0.998 & 0.997 & 0.998 & 0.338 & 0.293 & 0.281 & 0.373 & 0.327 & 0.281 \\
\hline Histogram equalization & -0.997 & -0.995 & -0.994 & -0.997 & -0.994 & -0.995 & -0.997 & -0.995 & -0.994 \\
\hline DCT compression & 0.999 & 0.999 & 0.999 & 0.759 & 0.721 & 0.716 & 0.557 & 0.509 & 0.474 \\
\hline
\end{tabular}


Table 2 shows the PSNR-HVS for various watermarked images using our proposed method. Table 3 illustrates the correlation coefficients of extracting watermarks with various attacks on watermarked image using the proposed watermarking scheme. Figures 10-17 illustrate the visual analysis of extracting watermarks with various attacks on watermarked image using our proposed watermarking scheme, FrWT and FrFT. From tables 2 and 3, figures 10-17, it is clear that the

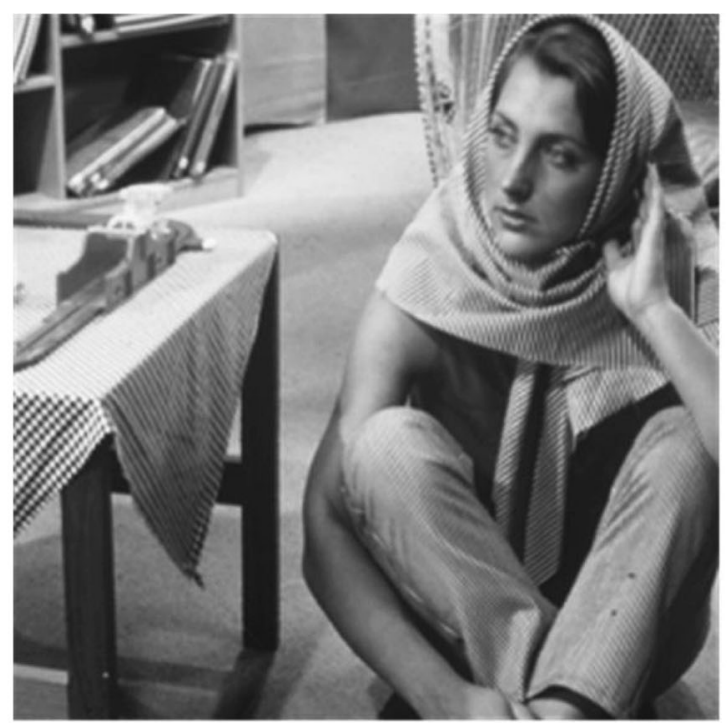

Host image

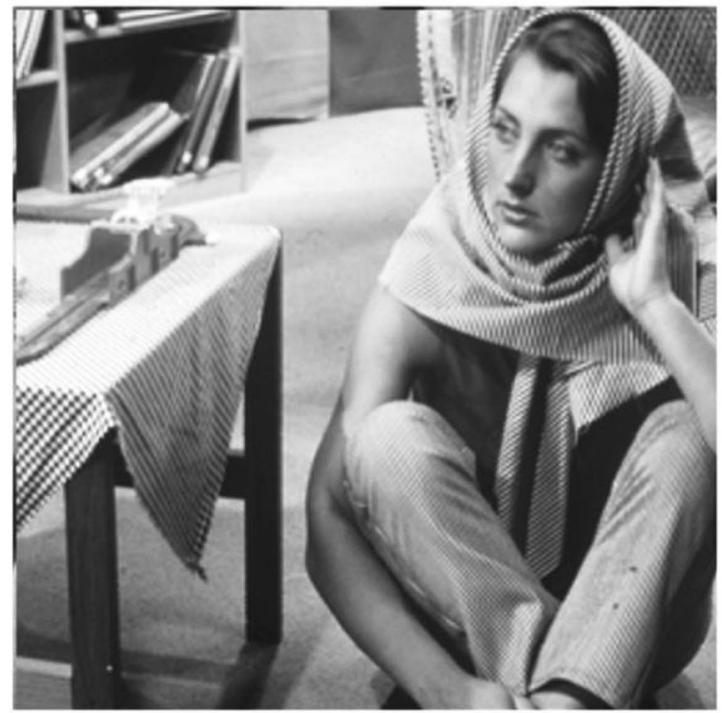

Watermarked image

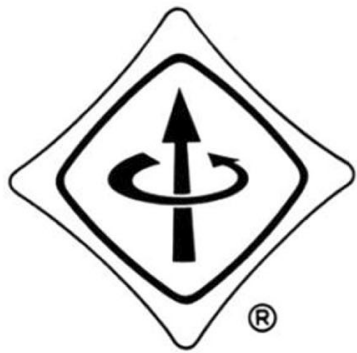

Watermark

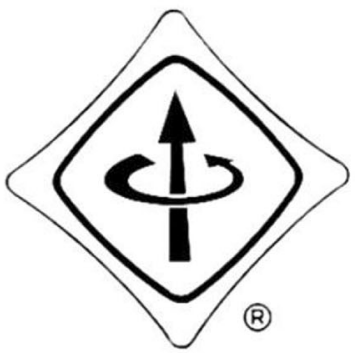

Extracted watermark

Figure 10. Results of watermarking by using proposed watermarking scheme. 


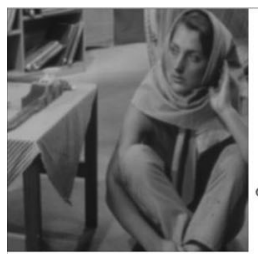

(a)

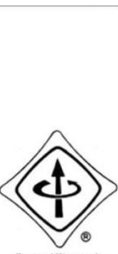

(b)

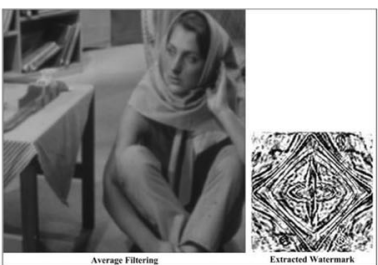

(c)

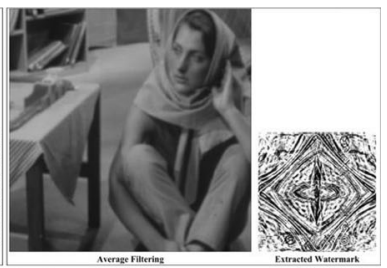

Figure 11. Extracted watermarks using: (a) Fr-M-band-DT-CWT, (b) FrWT and (c) FrFT with average filtering on watermarked image.

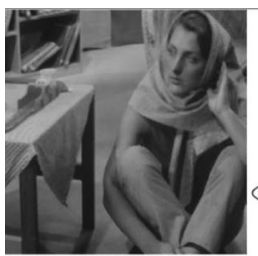

(a)

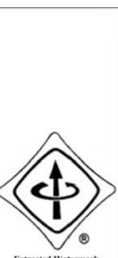

(b)

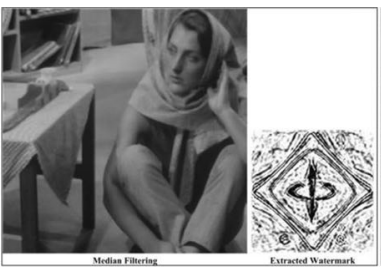

(c)

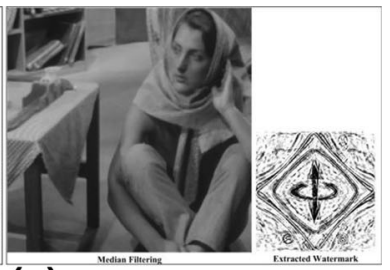

Figure 12. Extracted watermarks using: (a) Fr-M-band-DT-CWT, (b) FrWT and (c) FrFT with median filtering on watermarked image.

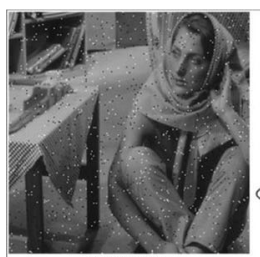

(a)

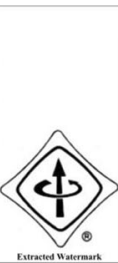

(b)

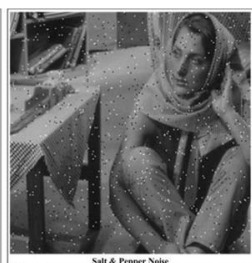

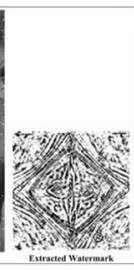

(c)

Figure 13. Extracted watermarks using: (a) Fr-M-band-DT-CWT, (b) FrWT and (c) FrFT with salt and pepper noise on watermarked image.

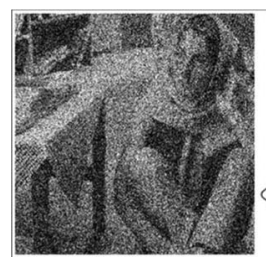

(a)

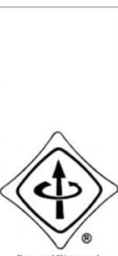

(b)

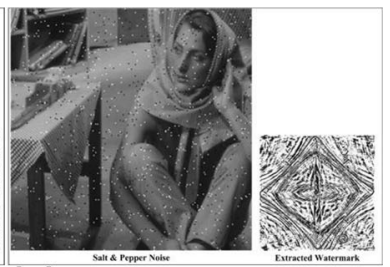




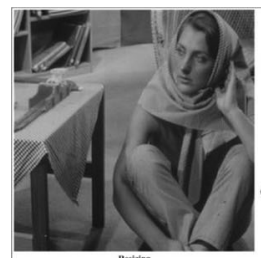

(a)

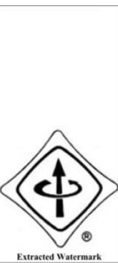

(b)

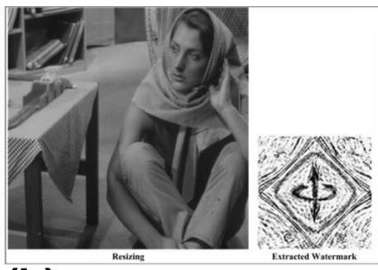

(c)

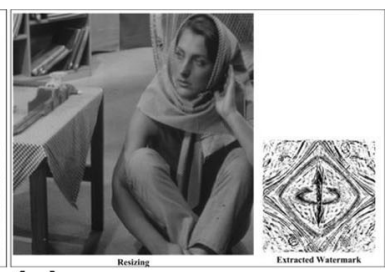

Figure 15. Extracted watermarks using: (a) Fr-M-band-DT-CWT, (b) FrWT and (c) FrFT with the resizing of watermarked image.

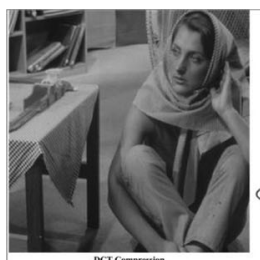

(a)

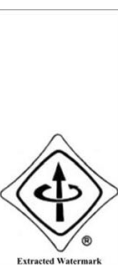

(b)

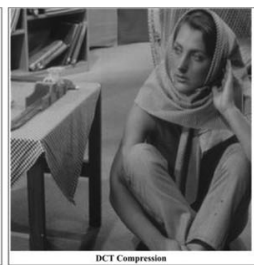

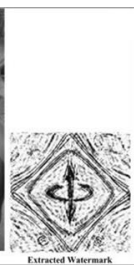

(c)

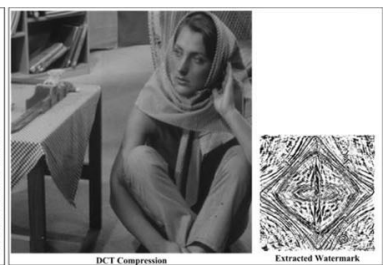

Figure 16. Extracted watermarks using: (a) Fr-M-band-DT-CWT, (b) FrWT and (c) FrFT with DCT compression on watermarked image.

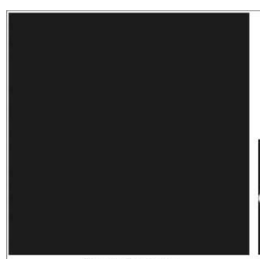

(a)

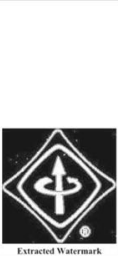

(b)

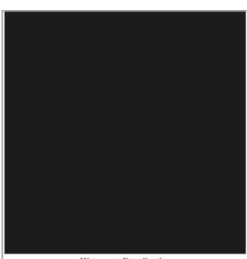

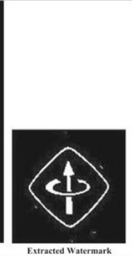

(c)

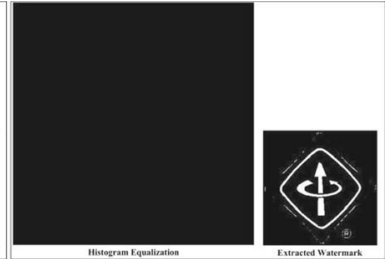

Figure 17. Extracted watermarks using: (a) Fr-M-band-DT-CWT, (b) FrWT and (c) FrFT with histogram equalization on watermarked image.

proposed watermarking scheme shows a significant improvement as compared to other existing watermarking schemes.

\section{Conclusions}

In this paper, we have proposed a novel logo watermarking scheme using fractional M-band dual-tree complex wavelet transform (Fr-M-band-DT-CWT). The combined concepts of M-band wavelets and DT-CWT i.e. M-band-DT-CWT has been employed for analyzing low frequency signal to enhance the performance. The performance of the proposed method is analysed with the three different host images and three different watermarks in terms of PSNR-HVS and correlation coefficient. The results of the investigations show that the proposed watermarking scheme is better as compared to other existing schemes with various attacks on the watermarked image. 


\section{References}

Adnan M Alattar, Eugene T Lin and Mehmet Utku Celik 2003 Digital Watermarking of Low Bit-Rate Advanced Simple Profile MPEG-4 Compressed Video. IEEE Transactions On Circuits And Systems For Video Technology 13(8): 1-3

Barni M, Bartiloni F, Cappellini V and Piva A 1998 A DCT Domain System for Robust Image watermarking. Signal Processing 66(3): 357-372

Chaux C, Duval L and Pesquet J C 2004 Hilbert pairs of M-band orthonotmal wavelet bases. In: Proc. Eur. Sig. and Image Proc. Conf.

Chin-Shiuh Shieh, Hsiang-Cheh Huang, Feng-Hsingwang and Jeng-Shyang Pan 2003 An Embedding Algorithm for Multiple Watermarks. J. Information Sci. Eng. 19: 381-395

Cox I J, Killian J, Leighton F T and Shamoon T 1997 Secure Spread Spectrum Watermarking for Multimedia. IEEE Transaction on Image Processing 6(12): 1673-1687

Daubechies I 1988 Orthonormal bases of compactly supported wavelets. Communications on Pure and Applied Mathematics 41: 909-996

Djurovic I, Stankovic S and Pitas I 2001 Digital watermarking in the fractional Fourier transformation domain. J. Network Computer Applications 24(4): 167-173

Dong Zheng, Jiying Zhao and Abdulmotaleb El Saddik 2003 RSTInvariant Digital Image Watermarking Based on Log-Polar Mapping and Phase Correlation. IEEE Transactions on Circuits Systems Video Technology 13(8): 1-14

Gaurav Bhatnagar and Balasubramanian Raman 2011 A new robust reference logo watermarking scheme. Multimed Tools Appl. 52: 621-640

Gopinath R A and Burrus C S 1992 Wavelets and filter banks. In: Wavelets: A tutorial in theory and applications, Chui C K (ed.) Academic Press, San Diego, CA, pp 603-654

Hwang M S, Chang C C and Hwang K F 1999 A watermarking technique based on one-way hash functions. IEEE Transactions on Consumer Electronics 45(2): 286-294

Jiang Xuehua 2010 Digital Watermarking and Its Application in Image Copyright Protection, International Conference on Intelligent Computation Technology and Automation Sch. of Eng., Linyi Normal Univ., Linyi, China, pp 114-117

Jixin Liu and Zheming Lu 2009 A Multipurpose Audio Watermarking Algorithm Based on Vector Quantization in DCT Domain, World Academy of Science. Eng. and Technol. 55: 618-623

Karen Egiazarian, Jaakko Astola, Nikolay Ponomarenko, Vladimir Lukin, Federica Battisti and Marco Carli 2006 A new full-reference quality metrics based on HVS, CD-ROM Proceedings of the Second International Workshop on Video Processing and Quality Metrics, Scottsdale, USA, 4

Kokare M, Biswas P K and Chatterji B N 2005 Texture Image Retrieval Using New Rotated Complex Wavelet Filters. IEEE Trans. Systems, Man, and Cybernetics 33(6): 1168-1178

Kundur D and Hatzinakos D 2004 Towards robust logo watermarking using meltiresolution image fusion. IEEE Transactions on Multimedia 6: 185-197

Mallat S 1989 A Theory for multiresolution signal decomposition: The wavelet representation. IEEE Trans. on Pattern Analysis Machine Intelligence 11(7): 674-693

Manesh Kokare, Biswas P K and Chatterji B N 2004 Cosine-modulated wavelet based texture features for content-based image retrieval. Pattern Recognition Lett. 25: 391-398

McBride A C and Kerr F H 1987 On Namias's fractional Fourier transforms. IMA J. Appl. Math. 39(2): $159-175$

Namias V 1980 The fractional order Fourier transform and its application to quantum mechanics. IMA J. Appl. Math. 25(3): 241-265

Prayoth Kumsawat, Kitti Attakitmongcol and Arthit Srikaew 2007 A Robust Image Watermarking Scheme Using Multiwavelet Tree. World Congress on Eng. 1: 612-618

Rioul O and Veterli M 1991 Wavelets and signal processing. IEEE Signal Processing Magazine 8: 14-38

Tetsuya Kojima, Naoki Ohtani, Takahiro Matsumoto and Udaya Parampalli 2011 A Blind Digital Watermarking Scheme Based on Complete Complementary Codes, Communications Theory Workshop (AusCTW), Univ. of Melbourne, Melbourne, VIC, Australia, pp 1-6 
Wang S H and Lin Y P 2004 Wavelet tree quantization for copyright protection watermarking. IEEE Transactions on Image Processing 13(2): 154-165

Xia X, Boncelet C G and Arce G R 1997 A multiresolution watermark for digital images. In: Proc. Fourth IEEE Int. Conf. on Image Processing, Santa Barbara, CA, 3, pp 548-551

Xinge You, Liang Du, Yiu-ming Cheung and Qiuhui Chen 2010 A Blind Watermarking Scheme Using New Nontensor Product Wavelet Filter Banks. IEEE Transactions On Image Processing 19(12): 3271-3284

Yu F Q, Zhangi Z K and Xu M H 2006 A Digital Watermarking Algorithm for Image Based on Fractional Fourier Transform. In: Proc. of First IEEE Conf. on Industrial Electronics and Applications, pp 1-5

Yuxin Liu, Bin Ni, Xiaojun Feng and Edward J Delp 2006 Lapped-orthogonal-transform-based adaptive image watermarking. J. Electronic Imaging 15(1): 013009-1-9

Zhang X D, Feng J and Lo K T 2003 Image watermarking using tree-based spatial-frequency feature of wavelet transform. J. Visual Comm. Image Representation 14: 474-491

Zou H and Tewfik A H 1992 Discrete orthogonal M-band wavelet decompositions. In: Proceedings of Int. Conf. on Acoustic Speech and Signal Processing, 4, pp IV-605-IV-608 